\title{
A Linked-Cell Domain Decomposition Method for Molecular Dynamics Simulation on a Scalable Multiprocessor
}

\author{
L. H. YANG, E. D. BROOKS III, AND J. BELAK \\ Massively Parallel Computing Initiative, Laurence Livermore Vational Laboratory, Litermore. C4 94.551
}

\begin{abstract}
A molecular dynamics algorithm for performing large-scale simulations using the Parallel C Preprocessor (PCP) programming paradigm on the BBN TC2000, a massively parallel computer, is discussed. The algorithm uses a linked-cell data structure to obtain the near neighbors of each atom as time evolves. Each processor is assigned to a geometric domain containing many subcells and the storage for that domain is private to the processor. Within this scheme, the interdomain (i.e., interprocessor) communication is minimized. (1) 1993 by John Wiley \& Sons, Inc.
\end{abstract}

\section{INTRODUCTION}

The molecular dynamics (IID) computer simulation method 1$\}$ is a well-established and important tool in the fields of phrsics. chemistry. biology. and engineering. Recent advances in computer hardnare and sofiware techniques have allowed the simulation of large sistems [2] using realistic potential models $[3$. + . Civen that the requirement for larese-scale and more realistic IID simulations will remain. the demand for advanced hardware and software architecture will continue to grow into the future. In anticipation of this liuture demand. we present an algorithm for per-

Recoived Februar: 1902

Revised Octuber jog.2

(c) 1993 hy John Wiley de Sons. Inc.

Scientific Programminir. Vol. 1. pp. 13:3-101:1992

CC. $1058-9 \cdot 2+4+93 / 02015 ; 3-009$ forming large-ncale YD simulations of simple metals with short-range embedded-atom interatomic potentials. on a scalable multiprocesons. the BBD TC:2000. The eurrent enentiguration of the $T(2000$ at Lawrence Livermore Pational Laboratory LLNL consists of 128 Monorola 88100 reduced instruction set microppocestors rumning at 20 $.11 \mathrm{~Hz}$. Earh processor is located on a separate board. or node. along with a 16-kilobre carbe and 16 megables of local memory. The nodes are interonnected by a sabable switch. At boot time. some of each procesonor s local memory is dedicated to an interleaved shared memory pool. Thus. allocated memory is either private accessible by only one processor making a memory reference within its onn node or shared accessible by all procesons via a memory reference through the switchi.

The method of YD simulation involves the evaluation of the force acting on each atom due 10 other atoms and the numerical intergation of the 
newtonian equations of motion. The most timeconsuming portion of any MD simulation is the evaluation of the force; it typically consumes up to $98 \%$ of the total simulation time. In general each atom interacts with every other atom in the system. However, for many physical systems such as rare gases and simple metals, the interaction is non-negligible for neighboring atoms within a few atomic diameters only and the computational demands are drastically reduced. Given that the neighbors of an atom do not change appreciably during a short period of simulation time, two general methods have been developed to keep track of an atom's neighbors. In the neighbor-list approach [5], all atoms within a sphere surrounding a given atom are stored in a list. The radius of the sphere is chosen to exceed the interaction range by an amount sufficient to permit updating of the neighbor list at intervals of several YID time-steps only (typically, 10-20 steps). The neighbor lists provide a natural mechanism for developing vectorized MD algorithms [6. However the large memory overhead associated with maintaining these lists prohibits usage in large-scale simulations $\left(>10^{6}\right.$ atoms). In the linked-cell methods. the system is subdivided into many small cells and linked-lists [?] of atoms belonging to each individual cell are constructed. Because of the random addressing of memory. linked-list algorithms do not vectorize efficiently and extensive effors have gone into vectorizing large-scale MD algorithms [8]. The number of atoms per linked cell depends on the interaction range and the software architecture whether the interaction is limited to neighboring subcells only or further subcells are allowed to interact). Lnlike the neighbor-list approach, the cell lists need to be renewed at each IID time-step. The low memory overhead associated with the linked-list is ideal for large-scale .ID simulation and the cell-list algorithm introduces a "natural" domain decomposition parallelism into the MD problem [9j. In this paper. we describe our effort to adapt this domain decomposition scheme to our linked-cell MD code $[10]$ in order to improve the parallel efficiency on the BBN TC2000. This scheme also allows for easy porting to machines supporting the message passing programming model only.

In general, a three-dimensional system of $\mathrm{N}$ atoms of mass $m$ in a simulation cell of sides $L_{x} . L_{y}$. and $L$, is the basic building block of an IID system. We employ an embedded-atom method (EA.M) [3] to express the interaction between the atoms in a simple metal. The total potential energy is written as:

$$
\tau_{\text {triml }}=\frac{1}{2} \sum_{i, j \neq i} \varphi\left(\mathrm{r}_{i j}\right)+\sum_{i} \mathrm{~F}\left(\rho_{i}\right)
$$

with

$$
\rho_{i}=\sum_{j \neq i} \mathrm{f}^{\prime}\left(\mathbf{r}_{i j}\right)
$$

The first term is the usual to-body interaction energy and the second term $\left(F\left(\rho_{i}\right)\right)$ is the energy required to embed the atoms into the local electronic charge density $\left(\rho_{i}\right)$, which is written as a superposition of charge densities due to neighboring atoms $\left(f\left(r_{i j}\right)\right.$. The nentonian equations of motion for the EAII are

$$
\begin{aligned}
m \frac{d^{2} \mathbf{x}_{k}}{d \mathrm{t}^{2}}= & -\sum_{j \neq \kappa}\left\{\varphi^{\prime}\left(\mathbf{r}_{k j}\right)+\left\langle\mathrm{F}^{\prime}\left(\rho\left(\mathbf{r}_{j}\right)\right\}\right.\right. \\
& \left.+\mathrm{F}^{\prime}\left(\boldsymbol{\rho}\left(\mathbf{r}_{k}\right)\right) \mathbf{f}^{\prime}\left(\mathbf{r}_{k j}\right)\right\} \frac{\mathbf{x}_{k}-\mathbf{x}_{j}}{\mathbf{r}_{k j}}
\end{aligned}
$$

These equations are inherently nonlocal: they depend on both the embedding densities at atom$k\left(\rho\left(r_{k}\right)\right)$ and at atom-j $\left(\rho\left(\mathrm{r}_{j}\right)\right)$. They must be solved in a two-step manner. The embedding density at all atomic sites $r_{j}$ is evaluated first. then the forces acting on each atom may be calculated.

The equations are integrated by approximating the time derivative with a central difference $[5$ :

$$
\frac{d^{2} x}{d t^{2}} \approx \frac{x^{\prime} t+\Delta t-2 x t+x / t-\Delta t}{\Delta t^{2}}
$$

where the time-step $(\Delta t)$ is $1 / 25$ of the vibration period $\left(\tau_{\mathrm{F}}\right)$. For a simple metal $\tau_{\mathrm{r}}$ is about $0.3 \times$ $10^{-12}$ seconds.

Domain decomposition techniques have received much attention recenty as they are suited for the parallel implementation of problems with localized data. The local nature of the short-range potential provides a good opportunity to apply the domain decomposition scheme to our MD simulation on a scalable multiprocessor machine. In greneral the physical YD cell is divided into geometrically separate subdomains. each containing many subcells. Each processor is assigned to a subdomain and is responsible for updating all at- 
oms contained therein. We employ the shared memory on the TC2000 as a "hub" for data communication berween subdomains, although the same communication/decomposition algorithm may also be implemented using the messagepassing programming model on distributed memory machines $[11-13]$.

The outline of this paper is as follows. In Section 2 we describe the basic data structures in our MD code and the programming model used on the BBN TC2000. In Section 3 we discuss our implementation of the linked-cell list method and the domain decomposition scheme on the BBN TC2000. Performance benchmark results are presented and discussed in Section 4.

\section{THE MD PROGRAM AND THE PARALLEL C PREPROCESSOR (PCP) PROGRAMMING MODEL}

Our ID program is designed to study rarious types of tribological systems fe.g. friction and wear). Details concerning the applications of MD simulations to these problems can be found in the papers by Belak and Stowers $[1+15 j$. Figure 1 illustrates a schematic geometry of a rypical system. For the simulation of the orthogonal metal cutting process. the MD simulation cell is a fixed window in the reference frame of the tool. The

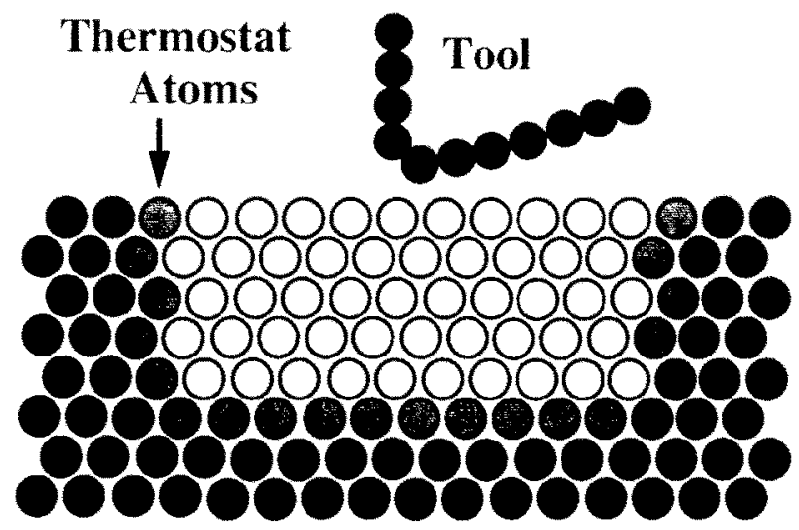

\section{Boundary Atoms}

FIGLRE 1 The geometry of our steady-state variableparticle molecular drnamics model of erthogonal metal cutting. The calculation is performed within the reference frame of the toel. The thermostat atoms are maintained at room remperature and the boundary atoms are used to impose the cutting speed-ther propagate to the right at the cutting speed. boundary atoms are used to impose the cutting speed; they move to the right at the cutting speed. In order to produce a steady state flow, new atoms are continuously inserted from the left, while atons that leave the top or the right of the cell are discarded: the system is open. Next to the boundary, we place a thermostat region. A time-dependent viscous damping $(\zeta)$ is added to the equation of motion for the atoms in this region $[16,17]$.

$$
m \frac{d^{2} \mathbf{x}_{k}}{\mathrm{dt}^{2}}=\text { force }-\zeta m \frac{\mathrm{d} \mathbf{x}_{k}}{\mathrm{dt}}
$$

with

$$
\frac{d \zeta}{d t}=\frac{1}{\tau^{2}}\left(\frac{T_{\text {rutc }}}{T_{\text {tesired }}}-1\right)
$$

Where $\tau$ io the relaxation time for $\zeta$ and $T$ atc is the kinetic temperature of the system. The purpose of the thermostat is to remove heat from doing work at the tool tip.

Our MD computer simulation code is written in the $\mathrm{C}$ programming language. Lnlike Fortran. C provides the advantages of allowing complex data structures, pointer arithmetic. and dynamic allocation of memory, all of which are necessary for performing variable atom simulations. We employ the PCP [18] as our programming model on the BBN TC2000. PCP provides an extension of the single-program-multiple-data (SPIID) programming model in the familiar $\mathrm{C}$ programming environment. Each processor executes the same code and the path through that code is determined by the data that the processor encounters. PCP introduces the concept of a "team" of processors. Each team has one master processor that is used for performing serial work such as data accumulation and initialization. Flow synchronization is ubtained through the barrier statement. Every processor reaching a barrier waits unil all members of its team (including the master) reach that barrier. Additional flow control for critical sections is accomplished with locks. PCP provides the lock (\&lock variable) and unlock (\&lock variable) functions to isolate critical sections. The lock variable is stored in shared memory. The first processor entering the critical section sets the lock variable to locked and proceeds with the calculation. Meanwhile, the remaining processors test the lock rariable to see whether it is locked. When the first processor finishes the calculation. it sets the lock variable to unlocked. The next pro- 
cessor to find it unlocked inmediately locks it and proceeds with the calculation.

Parallelism is exploited via domain decomposition. Each processor does the work for its domain and the interprocessor communication is performed through the shared memory. In effect. each processor is performing a separate MD simulation and obtaining boundary data from neighboring processors. Locality of data is exploited by explicitly declaring variables as private or shared with the private and shared storage class modifiers. Private and shared memory are dynamically allocated using the prmalloc and shmalloc func-tions.

The majority of our parallel MD code consists of routines from a standard serial MD code based on the linked-cell method. We use the linked-cell method in our parallel domain decomposition. Before going into our domain decomposition implementation. we list the most important functions in our VID code as follows:

1. Initialization

(a) Read input file

(b) Initialize positions and velocities build the linked-cell lists

2. Impose the domain decomposition scheme to divide the work for each processor

3. Main simulation loop

(a) force-calculate interatomic forces

(b) update-obtain new positions using the central difference

(c) kinetic-calculate kinetic energy and the temperature of the system

(d) output-accumulate data and output

(e) celler-apply boundary conditions and update linked-cell lists

The initialization and output blocks represent a small amount of serial work that is performed by the master processor. The heart of the work throughout the program consists of loops of the following type:

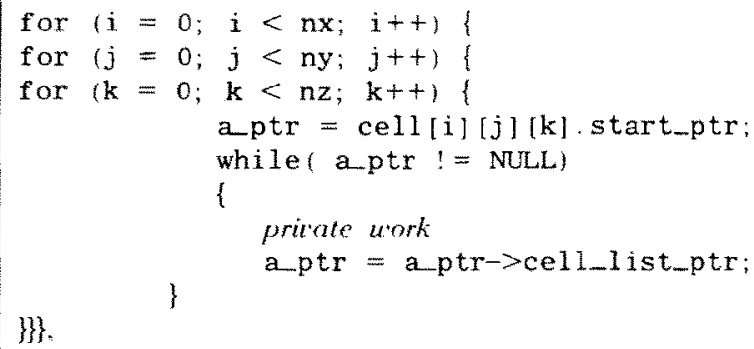


a)

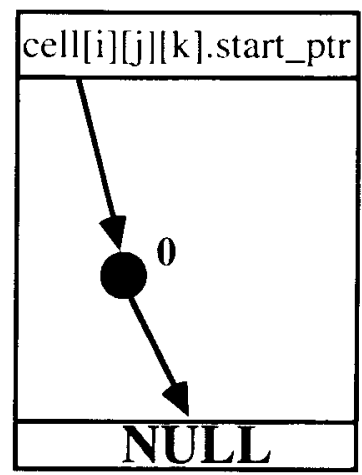

b)

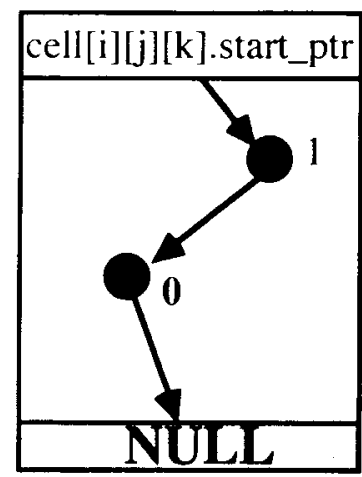

FIGLRE 2 The assignment of the firmt two atoms to the coll-linked list. Each atom calculates its cell indices according to the atom position. a The first atom is assigned to

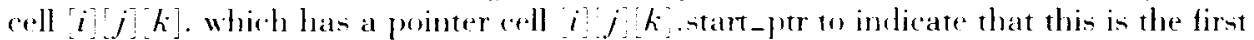
atom on the list. In addition. this atom is linked to NLLL. which is the parameter to indicate that this is the end of the cell line. b) The serond atom is added to the list by assigning it to the top of the list. By doing that the printer cell $i j j k$. start-ptr has been replaced by the second atom pointer and this atom in linked to the first atom.

has a pointer to the first atom it contains and has a flag to indicate the last atom it contains Fig. 2). Because the cell lists are built locally in each processor. critical sections are eliminated. Critical sections were eliminated from the previous version of the code $[10]$ by calculating both the force on atom- $i$ due to atom-j and the force on atom-j due to atom- $i$. thus doubling the work to assure scalability. At the end of the time-step. any atom that migrates from one cell to another cell is removed from its cell list and is added to another cell list. In Figure 3. we illustrate how an atom is removed from the middle of a cell list and is then added to the beginning of another cell list.

\subsection{Domain Decomposition}

In the domain decomposition scheme. the physical domain is divided into geometrically separate subdomains. each handled by a different processor. With the aim of minimizing the need for processors to communicate with one another and in order to obtain the necessary neighbor-cell information for the force calculation. we have introduced overlapping regions (Fig. to the so-called exterior border subcells). As a result. each processor norks independently at each VD time-step to calculate forces acting on each atom. obtain new atomic positions, and obtain new cell lists for inte- a)

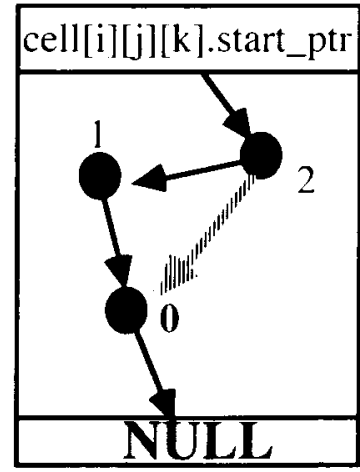

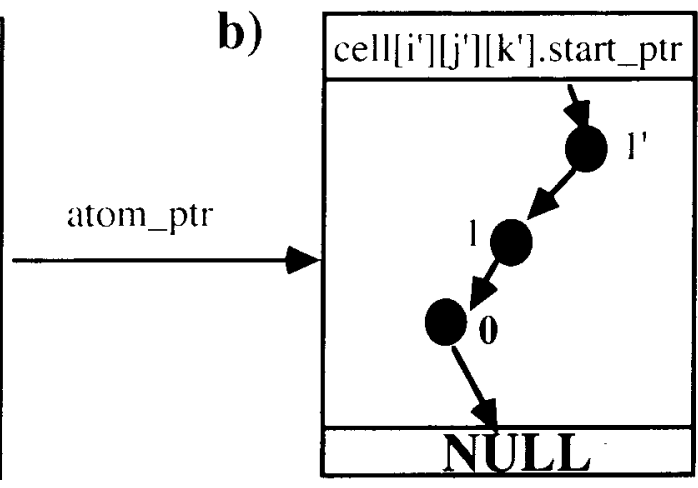

FIGURE 3 Cpdating the linked-cell list. Atom-1 is removed from the list by replacing the next atom pointer in atom- $2^{\prime}$ ' data snucture with the next atom pointer from atom1. Storage for atom-1 is returned to a buffer. b) Atom-1' is added to another cell list by connecting to the top of the list. Vote that the atom always carries its own pointer no matter where it goes. 


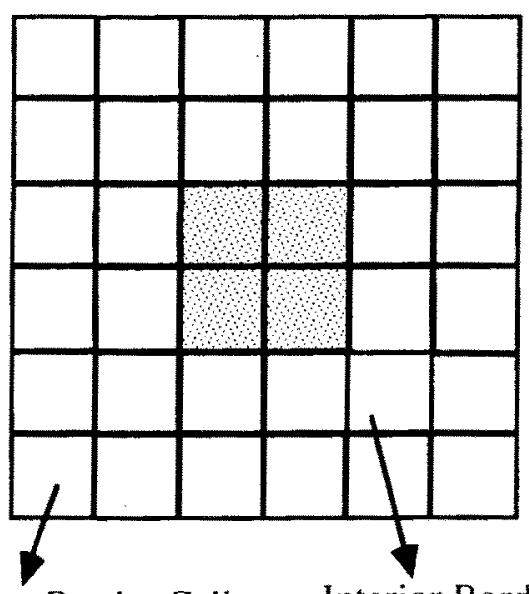

Exterior Border Cell Interior Border Cell

FIGURE 4 Molecular dynamics domain decomposition. At the beginning of an .ID simulation. each processor. _IPROC. is assigned to a set of linked-cells a domain). Each domain consists of a list of interior subcells (shaded squares) and exterior border subcell(empty squares). The assignment of exterior border subcells to each processor is for temporary storage used within the force calculation.

rior subcells. At the end of each time-step. interdomain communication takes place in order to update the data structure of the exterior border subcells.

The message-passing programming model may be used for performing the interdomain communication on distributed memory machines. like the TC2000 [12, 13]. Each processor knows the identity of its nearest neighbors. The data from the inner border subcells of each processor are sent to its neighboring processors. Each processor then receives messages from its neictubors and scatters the data appropriately to the outer border subcells. To ensure that the corner data are passed correctly, data are passed to neighboring processors in one direction first. then in another direction. The forces are calculated independently within each processor.

In our approach. we use the scalable shared memory facility of the BBN TC2000 to avoid the complexity of data packing and communicationsmanagement that would have been required by a strict message-passing implementation. To carry out the interdomain communication. we use the shared memory as a "hub" for temporary storage of new atomic positions in the border subcells. First, atomic positions of interior border subcells from each processor are copied into share memory. Then, each processor updates its exterior border subcells by copving those atomic positions from shared memory to local memory (Fig. 5). Finally, the linked-cell lists for each subdomain are renewed due to the migration of atoms from one subcell to another.

To make our procedure more instructive. we list four steps that are essential to this approach:

1. Cpdate all interior linked-cell lists.

2. Copy data from interior border subeells into shared memory.

3. Lpdate data in exterior border subcells by copying data from shared memory into private memory.

4. Lpdate linked-cell lists of interior and exterior border subcells.

Note that the linked-cell lists of the interior border subcells need to be renewed wice within this approach.

\section{PERFORMANCE RESULTS}

Our parallel VD alrorithm has been implemented on the BBY TC2000 using the PCP programming paradigm and we have performed some of the largest YD simulations for the longest periods of time to date. Here we present timing comparisons for three-dimensional EAII molecular dinamics simulations containing 4.032. 32.256. and $258.0+8$ atoms. All of our calculations are performed with $6+$ bit floating-point precision. Table 1 is a summary of the simulation parameters that define our YD sinulation. The benchmark calculation are performed for 20 IID time-stepi. though we have performed simulations for as long as several million time-steps. The timings from the lirst 10 time-steps are discarded and the last 10 time-steps are averaged to obtain C.PL times for one IID time-step.

On the BBN TC2000. the performance of the startup of a job is severely degraded by the thrashing of the virtual memory system as pare tables are constructed for each processor. This effect has been observed on other parallel systems implementing demand paged virtual memory and seems to be inherent in such srstems. Alihough the startup cost does not affect long problems that run for many thousands of time-steps. the chaotic timing effects that arise from the virual memory system during startup are very inconvenient when attempting to time portions of an application. 


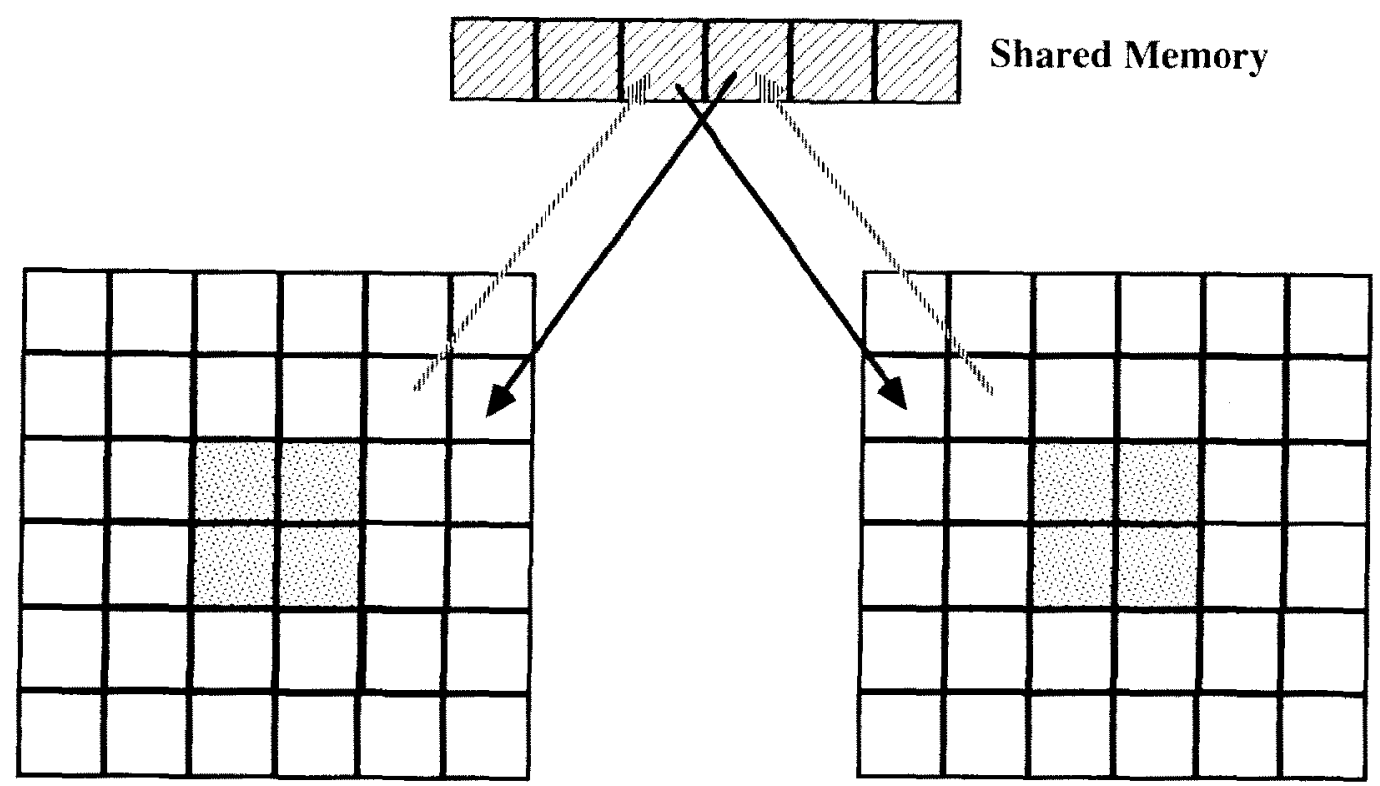

Processor I

Processor J

FIGLRE 5 The data communation through the shated memory. The data structure within each processor is updated in a two-step manter. The data from the interior border sulkells are copied to the shared memory. The updated data from the shared memory belonging to the exterior border subeells are then copied to acte processor.

Shown in Tables 2 and 3 are timings for the code executing on the TC2000 using the linkedcell domain decomposition scheme described in this paper. The present code executing on single processor uses memon local to the processor only-there is no shared memory overhead. The share memory is used for interprocesor communication only. There are about eight atoms per wubcell and each processor has a private cops of all data that define the calculation. The cel ler and io routines are the only routines that operate directly on shatred dala. From Table 3. we obsente

Table 1. Summary of Physical Variables in Our MD Simulation Presented in this Paper*

\begin{tabular}{ll}
\hline Parameter & Typical lalue \\
\hline Desired dimensionless temperature & \\
(T awsiret) & $0.5 i \varepsilon)$ \\
Density of the system & $0.9+21\left(\sigma^{-3}\right)$ \\
Relaxation time for $\zeta$ & 0.10 \\
Time-step in reduced units $(\Delta \mathrm{t})$ & $0.01(\tau)$ \\
\hline
\end{tabular}

* The E.M pottential parmeters were taken from lolian et al. 19 . The smulation state is a hot solid near the melting point. that the timings scale better than linear with system size. The ratio of the time spent doing communication to the time spent doing computational work is decreasing with increasing system size.

The main goal of this research was to adapt the domain decomposition scheme into the linkedcell MD code [10 which extensively utilized the shared memory during the MD simulation. although computationally intensive tasks were performed on local data only. It is therefore interesting to compare the limings for these two codes. In Table t. we show the timing comparisons of these

Table 2. CPU Times (Seconds) to Simulate One MD Time-Step of a Three-Dimensional Material Containing 32.256 Atoms on the BBN TC2000 (The Entire MD Simulation Box is Divided into 4.096 Subcells)

\begin{tabular}{lccc}
\hline Processors & 1 & 8 & 64 \\
\hline Total & 287.04 & 33.58 & 3.76 \\
force & 280.22 & $31.8+$ & 3.15 \\
kinetic & 0.96 & 0.13 & 0.03 \\
celler & 4.53 & 1.25 & 0.52 \\
iont others & 1.33 & 0.36 & 0.06 \\
\hline
\end{tabular}


Table 3. Total CPL Times (Seconds) to Simulate One MD Time-Step of a Three-Dimensional Material Containing 4,032, 32,256, and 258,048 Atoms on the BBN TC2000

\begin{tabular}{ccrr}
\hline Processors & 1 & 8 & \multicolumn{1}{c}{$6+$} \\
\hline 4.032 & 25.56 & 3.29 & 0.56 \\
32,256 & 287.04 & 33.58 & 3.76 \\
258,048 & 2367.296 & 209.35 & 28.22 \\
\hline
\end{tabular}

two codes running simulations of $4,032,32.256$. and $258.0+8$ atoms on $6 \pm$ processors. We obtain an average of threefold speed-up by using the domain decomposition scheme. Most of this performance increase is atributable to the increase in performance of the force routine. Foremost. we have decreased the computational work by a factor of two. Furthermore, we have minimized the interprocessor communication by confining the computational task to local memory

Figure 6 is a plot of the parallel efficiency of various routines in our code simulating the motion of 32.256 atoms. The parallel efficiency $(\eta)$ is defined to be the time to execute on one processor $\left(t_{1}\right)$ divided by the time to execute on $n$ processors $\left(t_{n}\right)$ divided by the total number of processors. That is:

$$
\eta=\frac{t_{1}}{t_{n} x n}
$$

The most inefficient routine in our code is the celler routine where border subcell information is copied to/from shared memory. The secondmost inelficient section of the code is the io routine, which we performed once at every time-step in the timings presented here. In a production run.

Table 4. Total CPU Times (Seconds) and Speed-Ups to Simulate One MD Time-Step of a Three-Dimensional EAMl Material Containing $4,032,32.256$, and 258,048 Atoms on the BBN TC2000 of 64 Processors: The Timing Results Are Based on the Same Initial Conditions and Physical Parameters for Both MD Codes and Obtain Identical Physical Results

\begin{tabular}{lcrr}
\hline Number of & & & \\
Atoms & +.032 & 32.256 & 258.048 \\
\hline Old & 1.62 & 11.89 & 93.56 \\
New & 0.56 & $3.7+$ & 28.2 .2 \\
Speed-up & 2.89 & 3.18 & 3.32 \\
\hline
\end{tabular}

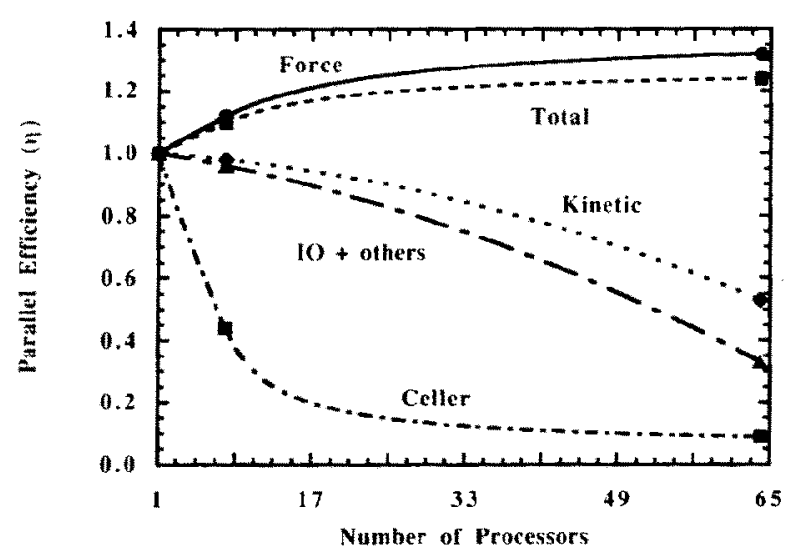

FIGURE 6 The parallel efliciency $(\eta)$ of the various routines in our IID code simulating 32.256 atoms in three-dimensional E.XI. The parallel efticiener $\eta$ is defined to be the time to execute on one processor divided by the time to execute on $n$ processors divided by the total number of processors.

io is executed every $~ 100$ time-steps and the effect on the overall performance is small.

The overall performance is somewhat surprising. providing an efficiency that is greater than 1 and reaching a platean of 1.2 at around $6+$ processors. Parallel efficiencies of greater than 1 are unusual, but have been documented before on multiprocessors employing cache equipped microprocessors as their basic compuational elements. There are essentially two places where this effect can arise, the first is in the cache used to store virtual to physical address translations and the second is in the cache used to store previousty referenced data. The BBN TC2000 is componed of processors possessing individual 16 Khyte data caches. and memory manasement units capable of mapping $50+K$ byte pages without TLB misses. As the number of procesiors is scaled. the total available cache memory seales as well. For a $32 \mathrm{~K}^{\circ}$ atom problem size. the heavily used portion of the data set begins to fit in the available carche memory as the processor count reaches $6+$ and no further speed increase results from the cache effect. This effect is important for all currently available microprocessors. but these microprocessors lack hardware monitors that nould allow us to pin down the source of the efficiency improvement in a more quantitative manner. We expect that future microprocessors will possess hardware monitors that are capable of counting cache misses and these will be very useful when tracking down anomolous performance results. 


\section{SUMMARY}

In this paper we have described a parallel implementation of our . YD coole using the linked-cell list method in conjunction with the domain decomposition approach on the BBN TC2000. Instead of using a message-pasing prouramming model for the interprocessor communication. we have emploved the available shared memory on the $T(2000$ as a hub for temporary data storage in the border subcells. We minimize interprocessor communication by contining the computational task to local memory. Nost of our performance increase is attributed to the increase in performance of the force routine. Our performance results have demonstrated that high parallel efficiency mas be obtained on the BBN $T(2000$ nith the PC.P programming model. We have obtained roughly three times the performance of the origrinal parallel version of the code on the $B B N$ $T(2000$ that did not une the domain decomponition technique. Lsing the domain decomposition technique on $6+$ processors of the BBN TC2000. we have exceeded o vimes the performance of a partially vecorized version of the algorithm on a single Cray XIIP procesonor. The partially vectorized version generates neighbor-lists from the linked-lists. similar to the algorithm of Grest et al. [8. It is clear that. for large-scale molecular dynamies algorithms with short-range forces. the masively parallel systems compete very favorably with comventional vector supercomputing technolory.

\section{ACKNOWLEDGMENTS}

This work was performed under the auspiess of the $L$.s. Deparment of Energy be the Lawrence Livermore $\mathrm{V}_{1-}$ tional Laboratory under eontract Vo. $11-7+05-\mathrm{E}$ VG$+8$

\section{REFERENCES}

[1] D. W. Hexmann. Computer Simulation . Methorls in Theoretical Phasics 2nd ed.l. Berlin: Springer-lerlag. 1990.

[2] W. G. Joover. A. J. DeGroot. C. G. Hower. 1. F. stowers. T. Kanai. B. L. Molian. T. Boku. S. lhara. and J. Belak. "Large-scale elastic-plastic indentation simulations via non-equilibrium molecular drnamics." Phass. Res. A. vol. +2. p. $58+4.1991$

[3] M. S. Daw and M. 1. Baskes. Fimbedded-atom method: derivation and application to impurities. surfaces. and other defects in metals." Phas. Ree. B. vol. 29. p. $0++3.198+$.

(4) J. Teroff. "Modeling solid-state chemistry: inter- atomic potentials for multicomponent stotems." Phys. Ree. B. val. 39. p. 5560. 1989 and references therein?

5) L. Verlet. "Computer 'experiments' on elasioal fluids. 1. Thermodynamie properties of LemnardJones molecules. " Plass. Rec.. wol. 1.59. p. 98. $196 ?$ ?

[6] D. Finchan and B. J. Ralston. "Mellecular dynamies simulation using the Cray-1 vector processing computer." Comput. Phass. Commun., vol. 23. p. 127. 1981.

[?] D. E. Knuth. The 1rt of Computer Prongemming: lolume I-Fundemental Algorithms. Readinger. MI: Adelison-ll estey. 1968.

8) G. S. (irest. B. Dunwer. and K. Kremer. "lectorized link cell FoRTR.AN colle for molecular dynamies simulations for a latere number of parti(les." (omput. Phas. Commun.. vol. 5.5. p. 269 ). 1989.

9) D. C. Rapaport. "Large-scale molecular dynamies simulation using vector and parallel computers." Comput. Phis. Rep.. vol. 9. p. 1. 1988.

10] J. Belak. The 199/ WPCI Hearly Report: The d1tack of the Niller Hicros. Livermore. Cit: Lawrence Livermore National Laboratom LC.RLID-107.02.2. 1991, p. 219.

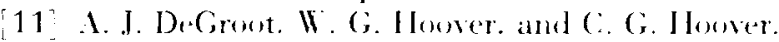
The 199l WPCI learly Report: The lleack of the hiller Micros. Livermore. C:A: Lawrence Livermore Vational Laboratory [C.RI-ID-107022. 1001. p. 211.

12] W. Smith. "Mrelecular druamics on hypercube parallel computers." Comput. Phas. Commun. wol. (6.2. p. 229. 1991.

[13? D. C. Rapaport. "Multi-million particle molecular dynamies Il. design considerations for distributed processing." Comput. Phas. Comman.. vol. 62. p. 217. 1991.

[1+ J. Bolak and I. F. Stowers. Macroscopic and Microscopic Processes. NATO 1SI Series. Serien E. Applied Science. Boston. MA: Kluwer Academic. 1902. p. 511 .

$15]$ J. Belak and I. F. Stowers. Proceedings of the International Conference on Metallargy Coatings and Thin Films. 1992. in press.

[16] S. Nose. "I unified formulation of the constant temperature molecular dynamics methods." $J$. Chem. Phys.. vol. 81. p. 511. 198t.

[17] W. C. Hoover. "Canonical dynamies: equilibrium phase space distribution." P'hys. Ret. A, vol. 31 . p. 1695.1985.

[18] E. D. Brooks. B. Gorda. and K. Warren. "The parallel C preprocessor." Scientific Programming. vol. 1. p. ?9. 1992.

[19] B. L. Holian. A. F. Loter. I. J. Wagner. R. J. Ravelo, S. P. Chen. W. G. Hoover. C. G. Hoover. J. E. Hammerberg. and T. D. Dontje. 'Effects of pairnise versus many-body forces on high-stress plastic deformation." Phys. Ret. A. vol. +3. p. 265.5. 1991 . 

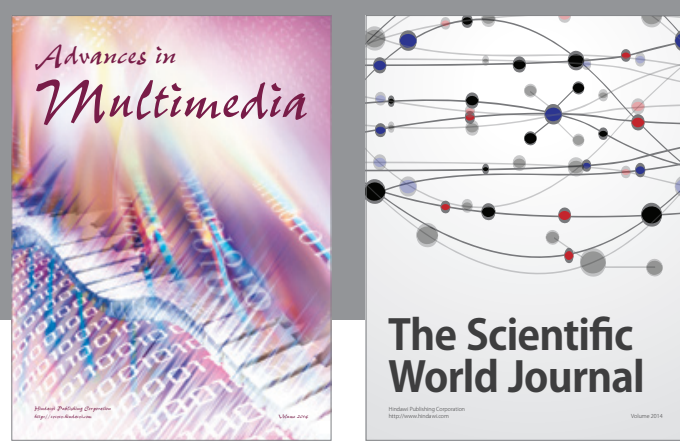

The Scientific World Journal
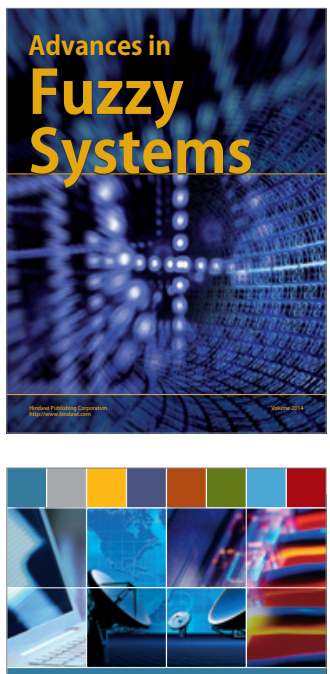

Computer Networks and Communications
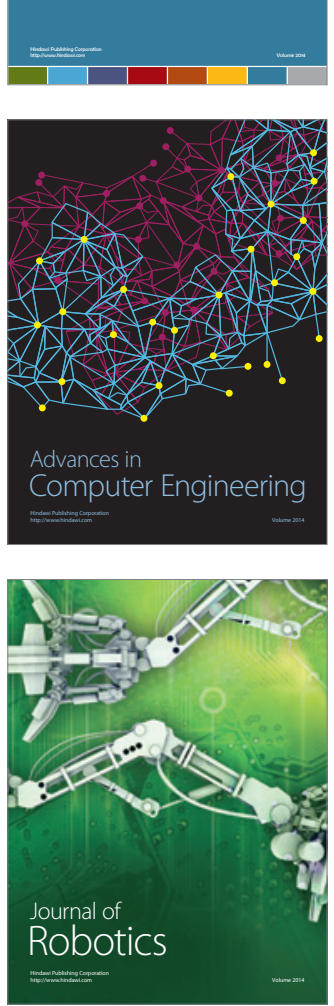
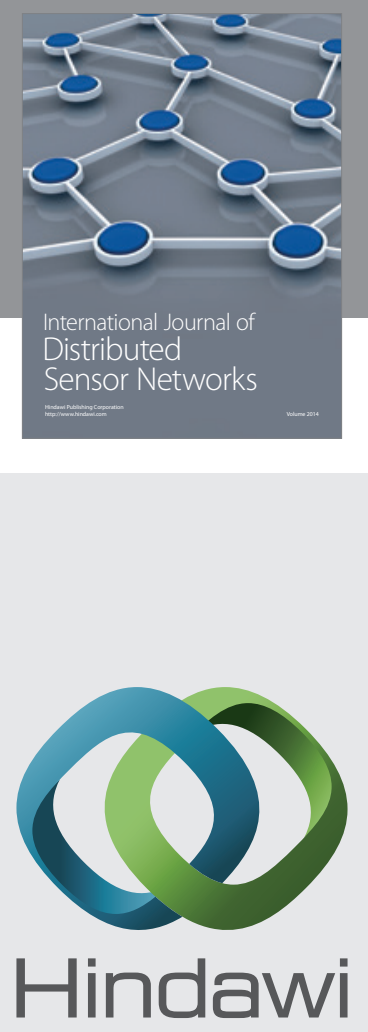

Submit your manuscripts at

http://www.hindawi.com
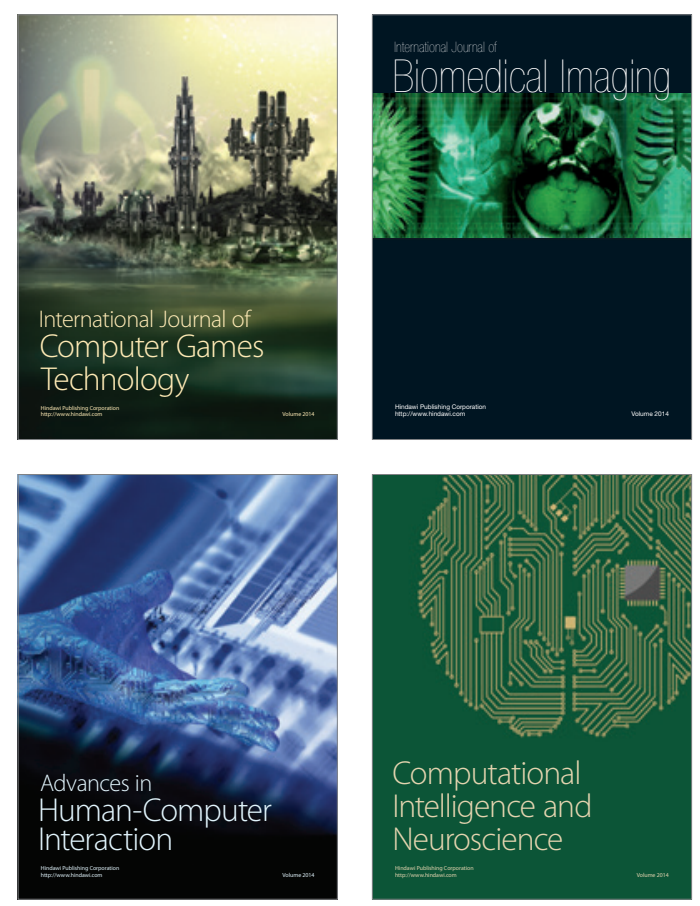
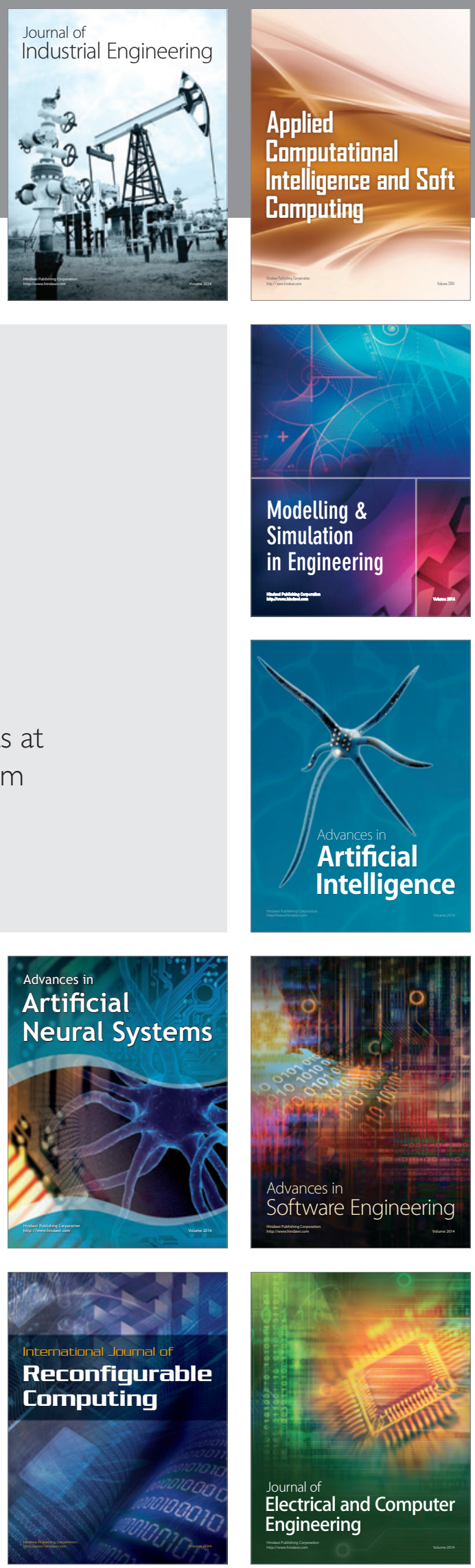